\title{
Active bodies in the near-Earth region: The tenuous boundary between comets and asteroids
}

\author{
Julio A. Fernández ${ }^{1}$ and Andrea Sosa ${ }^{2}$ \\ ${ }^{1}$ Departamento de Astronomía, Facultad de Ciencias, \\ Universidad de la República, Iguá 4225, 14000 Montevideo, Uruguay \\ email: julio@fisica.edu.uy \\ ${ }^{2}$ PDU Ciencias Físicas, Centro Universitario de la Región Este (CURE), \\ Universidad de la República, 27000 Rocha, Uruguay \\ email: asosa@cure.edu.uy
}

\begin{abstract}
We analyze the dynamics and activity observed in bodies approaching the Earth (perihelion distances $q<1.3 \mathrm{au})$ in short-period orbits $(P<20 \mathrm{yr}$ ), which essentially are near-Earth Jupiter Family Comets (NEJFCs) and near-Earth asteroids (NEAs). In the general definition, comets are "active", i.e. they show some coma, while asteroids are "inactive", i.e. they only show a bare nucleus. Besides their activity, NEJFCs are distinguished from NEAs by their dynamical evolution: NEJFCs move on unstable orbits subject to frequent close encounters with Jupiter, whereas NEA orbits are much more stable and tend to avoid close encounters with Jupiter. However, some JFCs are found to move on stable, asteroidal-type orbits, so the question arises if these objects are asteroids that have become active, perhaps upon approach to the Sun. In this sense they may be regarded as the counterparts of the main-belt comets (Hsieh \& Jewitt 2006). On the other hand, some seemingly inert NEAs move on unstable, comet-type orbits, so the question about what is a comet and what is an asteroid has become increasingly complex.
\end{abstract}

Keywords. comets: general; minor planets, asteroids

\section{Discussion}

Fernández \& Sosa (2015) studied the dynamical evolution of the observed NEJFCs during the last $10^{4} \mathrm{yr}$, and in some cases the integrations were extended to $5 \times 10^{4} \mathrm{yr}$ in the past. Whereas most comets move on highly unstable orbits as expected, they found that a small fraction of the sample comets move on stable, asterodal-like orbits over the computed time, very similar to the ones shown by most NEAs (Fernández et al. 2014). One of the methods to estimate the degree of stability of the orbit is to compute the time that the comet has stayed bound to the Sun in a short-period orbit with a perihelion distance smaller than $q_{o}+1 \mathrm{au}$, where $q_{o}$ is the perihelion distance of the comet at the discovery time. We show in Table 1 the list of NEJFCs whose orbits were found to be asteroidal by Fernández \& Sosa (2015). Their capture times in the near-Earth region $(q<1.3 \mathrm{au})$ are found to be $\gtrsim 5 \times 10^{4} \mathrm{yr}$, i.e. much longer than the typical lifetimes of NEJFCs.

We further analyze the different degrees of activity observed in near-Earth objects, which we represent through an equivalent fraction of active surface area $f$, defined as

$$
\begin{gathered}
f=\frac{Q_{H 2 O}}{4 \pi R^{2} Z} \\
142
\end{gathered}
$$


Table 1: NEJFCs in asteroidal orbits

\begin{tabular}{lll}
\hline Object & $R^{(*)}(\mathrm{km})$ & $\mathrm{t}_{\text {cap }}\left(10^{4} \mathrm{yr}\right)$ \\
\hline 66P /du Toit & 0.46 & $>5$ \\
162P/Siding Spring & 6.03 & $>5$ \\
169P/NEAT & 2.29 & $>5$ \\
182P/LONEOS & 0.38 & $>5$ \\
189P/NEAT & 0.60 & $>5$ \\
249P/LINEAR & $<1.20$ & 4.6 \\
300P/Catalina & 0.60 & $>5$ \\
P/2003 T12 (SOHO) & $<0.26$ & $>5$ \\
\hline
\end{tabular}

(*) The nucleus radii were computed assuming a geometric albedo $p_{V}=0.04$.

where $Q_{H_{2} O}$ is the production rate of gas (water) molecules, $R$ the radius of the body, and $Z$ the production rate of water molecules per unit area of a free-sublimating water ice surface (derived theoretically from the energy balance equation). Typical $f$ values of active NEJFCs are within a few percent to several tens percent (Fernández 2005). Yet observations of NEJFCs in asteroidal orbits lead to derived extreme low values of dust and/or gas production rates (Harmon et al. 2006; Kasuga et al. 2010; Schleichter 2014). The $f$ values should accordingly be much smaller than those derived for active comets, perhaps by one or more orders of magnitude, suggesting that NEJFCs in asteroidal orbits are much less active than typical JFCs. This may explain why the former can survive in the near-Earth region for much longer time scales than those of typical active comets of comparable size. These problems are addressed in this presentation.

\section{References}

Fernández, J. A. 2005, Comets. Nature, Dynamics, Origin, and their Cosmogonical Relevance (Springer, Dordrecht), p. 240

Fernández, J. A. \& Sosa, A. 2015, Planet. Space Sci., in press

Fernández, J. A., Sosa, A., Gallardo, T., \& Gutiérrez, J. N. 2014, Icarus 238, 1

Harmon, J. K., Nolan, M. C., Margot, J.-L., Campbell, D. B., Benner, L. A. M., \& Giorgini, J. D. 2006 , Icarus 184,285

Hsieh, H. H. \& Jewitt, D. 2006, Science 312, 561

Kasuga, T., Balam, D. D., \& Wiegert, P. A. 2010, Astron. J. 139, 1822

Schleicher, D. 2014, CBET No. 3881 